\title{
Dependencies among dependencies in matroids
}

\author{
James Oxley \\ Mathematics Department \\ Louisiana State University \\ Baton Rouge, Louisiana, USA \\ oxley@math.lsu.edu
}

\author{
Suijie Wang* \\ Institute of Mathematics \\ Hunan University \\ Changsha, Hunan, China \\ wangsuijie@hnu.edu.cn
}

Submitted: May 18, 2019; Accepted: Aug 18, 2019; Published: Sep 13, 2019

(c) The authors. Released under the CC BY-ND license (International 4.0).

\begin{abstract}
In 1971, Rota introduced the concept of derived matroids to investigate "dependencies among dependencies" in matroids. In this paper, we study the derived matroid $\delta M$ of an $\mathbb{F}$-representation of a matroid $M$. The matroid $\delta M$ has a naturally associated $\mathbb{F}$-representation, so we can define a sequence $\delta M, \delta^{2} M, \ldots$ The main result classifies such derived sequences of matroids into three types: finite, cyclic, and divergent. For the first two types, we obtain complete characterizations and thereby resolve some of the questions that Longyear posed in 1980 for binary matroids. For the last type, the divergence is estimated by the coranks of the matroids in the derived sequence.
\end{abstract}

Mathematics Subject Classifications: 05B35

\section{Introduction}

In algebraic topology, homology groups examine the independent holes of topological spaces. It is natural to ask about the dependence relations among these holes. For a 1dimensional simplicial complex (a graph), this amounts to determining the dependencies among all of the cycles in the graph.

As Judith Q. Longyear wrote in [5], at the Bowdoin College Summer 1971 NSF Conference on Combinatorics, Gian-Carlo Rota posed the following question: "The minimal dependent sets of vectors in a space $V$ may be regarded as vectors in the derived space $\delta V$ over the same field by using the vectors of $V$ as a basis for $\delta V$. Can this same sort of

\footnotetext{
*Supported by the National Natural Science Foundation of China (11871204)
} 
process be applied to the dependent sets of a matroid $M$ to investigate the 'dependencies among dependencies'? If so, what properties does $\delta M$, the derived matroid, possess?"

Longyear [5] answered the first question when $M$ is a binary matroid. She defined its derived matroid $\delta M$ to have as its ground set, the set $\mathcal{C}(M)$ of circuits of $M$ where a set $X$ of such circuits is independent in $\delta M$ exactly when, for each nonempty subset $Y$ of $X$, the symmetric difference of the circuits in $Y$ is nonempty. Longyear noted that her derived matroid is a binary matroid and she asked the following four questions about this matroid where we have differentiated the parts of these questions that contain more than one part.

- Question 1. (a) What effect does $\delta$ have on the flats of a matroid? (b) On the dual?

- Question 2. How many different (nonisomorphic) binary matroids $M$ are there for which $\delta M$ has rank $r$ ?

- Question 3. (a) When does $\delta M=M$ ? (b) When is there a matroid $N$ for which $\delta N=M$ ? (c) If $\delta^{k+1} M=\delta\left(\delta^{k} M\right)$, when can $\delta^{k} M=\delta^{j} M$ ?

- Question 4. If $M$ is $U_{1,3}$, then $\delta M$ is $U_{2,3}, \delta^{2} M$ is $U_{1,1}$ and $\delta^{3} M$ is $U_{0,0}$. Characterize those $M$ for which $\delta^{k} M$ can eventually be $U_{0,0}$.

In this paper, we answer Questions 3(a), 3(c), and 4 by proving the following results.

Theorem 1. Let $M$ be a binary matroid. If $\delta^{k} M \cong M$ for some $k \geqslant 1$, then $M \cong U_{0,0}$.

Theorem 2. Let $M$ be a binary matroid such that $\delta^{k} M \cong U_{0,0}$ for some $k \geqslant 0$. Then $\delta^{3} M \cong U_{0,0}$, and either $M \cong U_{0,0}$, or $M$ is nonempty and each if its components is isomorphic to $U_{1,1}$, a circuit, or the cycle matroid of a theta graph.

Also, we extend Longyear's work by defining the derived sequence $M, \delta M, \delta^{2} M, \ldots$ of matroids beginning with any $\mathbb{F}$-represented matroid $M$ where $\mathbb{F}$ is an arbitrary field. We show that, when $M$ has no coloops, $\delta M$ is connected if and only if $M$ is connected. Our main theorems show that derived sequences are either finite, cyclic, or divergent, and they answer Questions 3(a), 3(c), and 4 for arbitrary $\mathbb{F}$-represented matroids. In particular, we prove that $U_{2,4}$ is the unique nonempty connected matroid $M$ for which $\delta M \cong M$. These results appear in Section 4 of the paper. In Sections 2 and 3, we define derived sequences of represented matroids and prove a number of basic properties of derived matroids.

\section{The derived sequence of a represented matroid}

Our matroid terminology and notation will follow Oxley [6]. For a field $\mathbb{F}$, let $M$ be an $\mathbb{F}$-representable matroid with ground set $E=\left\{e_{1}, e_{2}, \ldots, e_{m}\right\}$, and let $\varphi: E \rightarrow \mathbb{F}^{n}$ be a representation of $M$. The matrix $A$ whose columns are the vectors $\varphi\left(e_{1}\right), \varphi\left(e_{2}\right), \ldots, \varphi\left(e_{m}\right)$ is the matrix corresponding to $\varphi$. Moreover, $M$ is $M[A]$, the vector matroid of the matrix $A$. The matrix $A$ is also referred to as an $\mathbb{F}$-representation of $M$. 
The pair $(M, \varphi)$, or equivalently the pair $(M, A)$, denotes an $\mathbb{F}$-represented matroid. For such a pair, associated with each circuit $C$ of $M$, there is a vector $\mathbf{c}_{C}=\left(c_{1}, c_{2}, \ldots, c_{m}\right)$ in $\mathbb{F}^{m}$ such that $\sum_{i=1}^{m} c_{i} \varphi\left(e_{i}\right)=0$ where $c_{i} \neq 0$ if and only if $i \in C$. Moreover, as one easily checks, $\mathbf{c}_{C}$ is unique to within a non-zero constant scalar multiple. It follows that, associated with the $\mathbb{F}$-represented matroid $(M, \varphi)$, there is an $\mathbb{F}$-represented matroid $(\delta M, \delta \varphi)$ with ground set $\mathcal{C}(M)$, the set of circuits of $M$, such that $(\delta \varphi)(C)=\mathbf{c}_{C}$ for all $C$ in $\mathcal{C}(M)$. We call the $\mathbb{F}$-represented matroid $(\delta M, \delta \varphi)$ the derived matroid of $(M, \varphi)$; the vector $\mathbf{c}_{C}$ is the circuit vector of $C$. We shall frequently write $\delta M$ for $(\delta M, \delta \varphi)$.

Let $\left(\delta^{0} M, \delta^{0} \varphi\right)=(M, \varphi)$. Inductively, for any positive integer $k$, the $k$ th derived matroid $\left(\delta^{k} M, \delta^{k} \varphi\right)$ of $M$ is the derived matroid of $\left(\delta^{k-1} M, \delta^{k-1} \varphi\right)$. The derived sequence of $(M, \varphi)$ is the sequence $\left(\delta^{0} M, \delta^{0} \varphi\right),\left(\delta^{1} M, \delta^{1} \varphi\right),\left(\delta^{2} M, \delta^{2} \varphi\right), \ldots$ Since over $G F(2)$, taking linear combinations of vectors coincides with taking symmetric differences of their supports, this definition is easily seen to extend Longyear's definition of derived matroids of binary matroids. For such matroids, Recski [7] denotes $\delta M$ by $\theta(M)$ and refers to the operation $M \rightarrow \theta(M)$ as the theta-operation on $M$.

For a field $\mathbb{F}$, let $(M, \varphi)$ be an $\mathbb{F}$-represented matroid and $A$ be the matrix corresponding to $\varphi$. Elementary row operations on $A$ clearly do not alter the circuit vectors of $(M, \varphi)$. This means that, when $r(M)>0$, for any ordered basis $\left(e_{1}, e_{2}, \ldots, e_{r}\right)$ of $M$, we can assume, after potentially permuting columns, that $A$ is a standard representative matrix $\left[I_{r} \mid D\right]$ for $M$, where $I_{r}$ is the $r \times r$ identity matrix and its columns are labelled, in order, $e_{1}, e_{2}, \ldots, e_{r}$.

For a basis $B$ in a matroid $M$ and an element $e$ of $E(M)-B$, the unique circuit $C(e, B)$ contained in $B \cup e$ is called the fundamental circuit of e with respect to $B$.

Lemma 3. For a field $\mathbb{F}$, let $(M, \varphi)$ be an $\mathbb{F}$-represented matroid. Then $\delta M$ is a simple matroid of rank $r^{*}(M)$. In particular, if $B$ is a basis of $M$, then $\{C(e, B): e \in E(M)-B\}$ is a basis of $\delta M$.

Proof. It is clear that $\delta M$ is simple since no circuit vector is the zero vector while no two distinct circuits have circuit vectors that are scalar multiples of each other. If $r(M)=0$, then $M \cong U_{0, m}$, so $\delta M \cong U_{m, m}$ and the result holds. Now suppose $r(M)>0$. As noted above, we can transform the matrix $A$ corresponding to the representation $\varphi$ into the form $\left[I_{r} \mid D\right]$. Let the columns of this matrix be labelled, in order, by $e_{1}, e_{2}, \ldots, e_{m}$ where $\left\{e_{1}, e_{2}, \ldots, e_{r}\right\}$ is a basis $B$ of $M$. For each $i$ in $\{1,2, \ldots, m-r\}$, consider $C\left(e_{r+i}, B\right)$. Clearly the $m-r$ circuit vectors of $C\left(e_{r+1}, B\right), C\left(e_{r+2}, B\right), \ldots, C\left(e_{m}, B\right)$ are linearly independent. As all of the circuit vectors of $M$ are in the solution space of the equation $\left[I_{r} \mid D\right] X=0$, we deduce that $\delta M$ has $\left\{C\left(e_{i}, B\right): r+1 \leqslant i \leqslant m\right\}$ as a basis. Thus $r(\delta M)=m-r=|E(M)|-r(M)=r^{*}(M)$.

For a basis $B$ of an $\mathbb{F}$-represented matroid $(M, \varphi)$, we shall call $\{C(e, B): e \in$ $E(M)-B\}$ the circuit basis of $\delta M$ associated with $B$. Because the fundamental circuits do not depend on the representation $\varphi$, this circuit basis also does not depend on the representation. 


\begin{tabular}{|c|c|c|c|c|c|c|c|}
\hline & 124 & 135 & 236 & 456 & 1346 & 1256 & 2345 \\
\hline$\varphi(1)$ & 1 & 1 & 0 & 0 & 1 & 1 & 0 \\
\hline$\varphi(2)$ & 1 & 0 & 1 & 0 & -1 & 0 & 1 \\
\hline$\varphi(3)$ & 0 & 1 & -1 & 0 & 0 & -1 & -1 \\
\hline$\varphi(4)$ & -1 & 0 & 0 & 1 & 0 & -1 & -1 \\
\hline$\varphi(5)$ & 0 & -1 & 0 & -1 & -1 & 0 & 1 \\
\hline$\varphi(6)$ & 0 & 0 & -1 & -1 & 1 & 1 & 0 \\
\hline
\end{tabular}

Figure 1: A matrix representation of $\delta M\left(K_{4}\right)$.

Corollary 4. For a field $\mathbb{F}$, let $(M, \varphi)$ be an $\mathbb{F}$-represented matroid. Then

$$
|\mathcal{C}(M)|=r^{*}(M)+r^{*}(\delta M) .
$$

The following matrix $A$ represents $M\left(K_{4}\right)$ over both $G F(2)$ and $G F(3)$ where, of course, $-1=1$ in the former:

$$
\begin{gathered}
1 \\
{\left[\begin{array}{cccccc}
1 & 2 & 3 & 4 & 5 & 6 \\
0 & 1 & 0 & 1 & 1 & 0 \\
0 & 0 & 1 & 0 & 1 & -1
\end{array}\right] .}
\end{gathered}
$$

We will write $A_{2}$ and $A_{3}$ for the interpretations of $A$ over $G F(2)$ and $G F(3)$, respectively. Hence we can view $M\left[A_{2}\right]$ and $M\left[A_{3}\right]$ as $G F(2)$ - and $G F(3)$-represented matroids.

Now $M\left(K_{4}\right)$ has exactly seven circuits. These label the columns of the matrix in Figure 1, where, for example, 124 is an abbreviation for $\{1,2,4\}$. Row $i$ of this matrix is labelled by the column vector $\varphi(i)$ corresponding to column $i$ in $A$. The columns of this matrix are the circuit vectors of the corresponding circuits. Thus $\delta M\left[A_{2}\right]$ and $\delta M\left[A_{3}\right]$ are represented by this matrix interpreted over $G F(2)$ and $G F(3)$, respectively. By Lemma $3, \delta M\left[A_{2}\right]$ and $\delta M\left[A_{3}\right]$ both have rank three. Clearly each is simple having seven elements. Thus $\delta M\left[A_{2}\right] \cong F_{7}$, the Fano matroid. Since $\delta M\left[A_{3}\right]$ is ternary, we deduce that $\delta M\left[A_{3}\right] \varsubsetneqq \delta M\left[A_{2}\right]$. It is not difficult to check that $\delta M\left[A_{3}\right] \cong F_{7}^{-}$, the non-Fano matroid.

In contrast to the above, where we considered representations of a binary matroid over two different fields, if we fix the field $\mathbb{F}$, then the derived matroid of a binary matroid does not depend on the representation.

Lemma 5. Let $M$ be a binary matroid and let $\varphi$ and $\psi$ be $\mathbb{F}$-representations of $M$ for some field $\mathbb{F}$. Then $(\delta M, \delta \varphi)=(\delta M, \delta \psi)$.

Proof. By a theorem of Brylawski and Lucas [1] (see [6, Proposition 6.6.5]), as $M$ is binary, all $\mathbb{F}$-representations of $M$ are projectively equivalent. Thus if $A_{1}$ and $A_{2}$ are the matrices corresponding to $\delta \varphi$ and $\delta \psi$, then there are non-singular matrices $X$ and $Y$, where $Y$ is diagonal, such that $A_{2}=X A_{1} Y$. It follows, by using determinants to compare the sets of bases, that $M\left[A_{2}\right]=M\left[A_{1}\right]$; that is, $(\delta M, \delta \varphi)=(\delta M, \delta \psi)$. 
The derived matroid of the smallest non-binary matroid, $U_{2,4}$, depends neither on the representation nor the field. More generally, we have the following result.

Lemma 6. For a field $\mathbb{F}$ and $n \geqslant 3$, let $\varphi$ be an $\mathbb{F}$-representation of $U_{n-2, n}$. Then $\delta U_{n-2, n} \cong U_{2, n}$. In particular, $\delta U_{2,4} \cong U_{2,4}$.

Proof. Clearly $\delta U_{n-2, n}$ has $n$ elements. By Lemma 3, this matroid is simple of rank two and so it is isomorphic to $U_{2, n}$.

The derived matroids of rank-one matroids are the cycle matroids of complete graphs.

Lemma 7. For a field $\mathbb{F}$ and $n \geqslant 1$, let $\varphi$ be an $\mathbb{F}$-representation of $U_{1, n}$. Then $\delta U_{1, n} \cong$ $M\left(K_{n}\right)$.

Proof. As $U_{1, n}$ is binary, by Lemma 5, we may assume that $\mathbb{F}=G F(2)$. Then $\delta U_{1, n}$ is represented over that field by the $n \times\left(\begin{array}{l}n \\ 2\end{array}\right)$ matrix whose columns are all distinct vectors of length $n$ having exactly two non-zero entries. This matrix also represents $M\left(K_{n}\right)$.

For a fixed field $\mathbb{F}$, we know that $\delta M$ does not depend on the $\mathbb{F}$-representation of $M$ when $M$ is binary. We now show that this does not hold in general.

Theorem 8. Let $\mathbb{F}$ be a field. Then, for all $\mathbb{F}$-represented matroids $(M, \varphi)$ the derived matroid $\delta M$ does not depend on the $\mathbb{F}$-representation $\varphi$ if and only if $\mathbb{F}$ is $G F(2)$ or $G F(3)$.

Proof. If $\mathbb{F}=G F(2)$, then, as noted above, $\delta M$ does not depend on the $G F(2)$-representation of $M$. Now let $\mathbb{F}=G F(3)$. Then, by a theorem of Brylawski and Lucas [1] (see [6, Corollary 14.6.1]), all $G F(3)$-representations of a ternary matroid $M$ are projectively equivalent. Hence $\delta M$ does not depend on the representation. For the converse, we use two examples.

We view the field $G F(4)$ as $G F(2)(\omega)$ where $\omega^{2}+\omega+1=0$. Kahn [3] noted that $U_{2,4} \oplus_{2} U_{2,4}$ is represented over $G F(4)$ by the matrix $A_{x}$ for each $x$ in $\{\omega, \omega+1\}$ where

$$
A_{x}=\left[\begin{array}{llllll}
1 & 2 & 3 & 4 & 5 & 6 \\
0 & 1 & 1 & 0 & 1 & 1 \\
1 & 1 & \omega & 0 & 0 & 0 \\
0 & 0 & 0 & 1 & 1 & x
\end{array}\right]
$$

Then the matrix representing $\delta M\left[A_{x}\right]$ has the following submatrix:

$$
\left[\begin{array}{ccc}
1246 & 1345 & 2356 \\
1 & \omega & 0 \\
1 & 0 & (\omega+1)(x+1) \\
0 & 1 & \omega(x+1) \\
x & 1 & 0 \\
0 & 1 & x \\
1 & 0 & 1
\end{array}\right] .
$$


These three columns are linearly dependent when $x=\omega+1$ but are linearly independent when $x=\omega$. Thus $\delta M$ may depend on the $\mathbb{F}$-representation of $M$ when $\mathbb{F}=G F(4)$.

For a field $\mathbb{F}$ with at least four elements, the matroid $U_{3,6}$ is represented by the matrix

$$
A=\left[\begin{array}{cccccc}
1 & 2 & 3 & 4 & 5 & 6 \\
1 & 0 & 0 & 1 & 1 & 1 \\
0 & 1 & 0 & 1 & x_{1} & x_{2} \\
0 & 0 & 1 & 1 & x_{3} & x_{4}
\end{array}\right]
$$

where $x_{1}, x_{2}, x_{3}$, and $x_{4}$ are elements of $\mathbb{F}-\{0,1\}$ such that

$$
\left\{x_{1}, x_{4}\right\} \cap\left\{x_{2}, x_{3}\right\} \neq \varnothing, \quad x_{1} x_{4} \neq x_{2} x_{3}, \quad \text { and } \quad\left(x_{1}-1\right)\left(x_{4}-1\right) \neq\left(x_{2}-1\right)\left(x_{3}-1\right) .
$$

We know that $\delta M[A]$ has fifteen elements and rank three and is spanned by the fundamental circuits of the elements 4,5 , and 6 with respect to the basis $\{1,2,3\}$ of $M[A]$. If we consider the matrix $\delta A$ whose columns are labelled by the circuits of $\delta M[A]$ and whose rows are labelled by the columns of $A$, we see that the submatrix of this matrix whose columns are labelled by these fundamental circuits, $\{1,2,3,4\},\{1,2,3,5\}$, and $\{1,2,3,6\}$, and whose rows are labelled by 4,5 , and 6 is $I_{3}$. It follows that $\delta M[A]$ is represented by the submatrix of $\delta A$ obtained by removing its first three rows. This submatrix has the following submatrix:

$$
\begin{aligned}
& \begin{array}{lll}
1246 & 1356 \quad 2345
\end{array} \\
& {\left[\begin{array}{ccc}
-x_{4} & 0 & -1 \\
0 & -x_{2} & 1 \\
1 & x_{1} & 0
\end{array}\right] .}
\end{aligned}
$$

As the determinant of this matrix is $x_{1} x_{4}-x_{2}$, it follows that $\delta M[A]$ does depend on the representation provided the field is large enough to allow us to choose two different 4 -tuples $\left(x_{1}, x_{2}, x_{3}, x_{4}\right)$ such that $x_{1} x_{4}-x_{2}=0$ for one of these 4 -tuples but $x_{1} x_{4}-x_{2} \neq 0$ for the other. As the reader can easily check, this is possible provided $\mathbb{F}$ has at least five elements. Thus, for all such fields, $\delta M$ may depend on the $\mathbb{F}$-representation of $M$.

The last result is not surprising since it is well known that all $\mathbb{F}$-representations of an $\mathbb{F}$-representable matroid are projectively equivalent if and only if $\mathbb{F}$ is $G F(2)$ or $G F(3)$.

Next we observe that adding an element in series to an existing element of $M$ does not alter $\delta M$.

Lemma 9. For a field $\mathbb{F}$, let $(M, \varphi)$ be an $\mathbb{F}$-represented matroid. If $\{e, f\}$ is a cocircuit of $M$, then $\delta(M / e)=\delta(M)$ where the representation of $M / e$ is that induced by $\varphi$.

Proof. As $M$ has $\{e, f\}$ as a cocircuit, it has a basis $B$ that contains $e$ but not $f$. We can transform the matrix $A$ corresponding to $\varphi$ into a matrix of the form $\left[I_{r} \mid D\right]$ where the columns of the identity matrix are labelled by the elements of $B$, and the first columns of $I_{r}$ and $D$ are labelled by $e$ and $f$, respectively. Then $M / e$ is represented by the matrix that is obtained from $\left[I_{r} \mid D\right]$ by deleting the first row and the first column. As $e \cup(E(M)-B)$ contains a unique cocircuit of $M$ and $\{e, f\}$ is a cocircuit contained in this set, the only 
non-zero entries in the first row of $\left[I_{r} \mid D\right]$ are in the columns labelled by $e$ and $f$. Hence a circuit of $M$ contains $e$ if and only if it contains $f$. Thus, in the representation of $\delta M$ in which the columns are the circuit vectors and the rows are labelled by the elements of $M$, the row labelled by $f$ is a non-zero scalar multiple of the first row, the row labelled by $e$. Thus deleting the row labelled by $f$ gives a representation for the same matroid. As $\left(c_{1}, c_{2}, \ldots, c_{n}\right)$ is a circuit vector of $M$ if and only if $\left(c_{2}, c_{3}, \ldots, c_{n}\right)$ is a circuit vector of $M / e$, we deduce that $\delta(M / e)=\delta M$.

The next lemma specifies what effect the presence of a loop or a coloop has on $\delta M$.

Lemma 10. Let $(M, \varphi)$ be an $\mathbb{F}$-represented matroid.

(i) If $e$ is a coloop of $M$, then $\delta M=\delta(M \backslash e)$.

(ii) If e is a loop of $M$, then $\delta M=U_{1,1} \oplus \delta(M \backslash e)$.

Proof. The first part is immediate from the fact that a coloop is in no circuits. Now suppose $e$ is a loop of $M$. The only circuit vector with a non-zero entry in the coordinate corresponding to $e$ is the circuit vector of the circuit $\{e\}$. Hence $\{e\}$ is a coloop of $\delta M$.

Recall that, for a matroid $M$, its cosimplification $\operatorname{co}(M)$ is the matroid that is obtained from $M$ by deleting all coloops and then contracting all but one element from each series class. The following result is an immediate consequence of the last two lemmas.

Corollary 11. For a field $\mathbb{F}$, let $(M, \varphi)$ be an $\mathbb{F}$-represented matroid. Then $\delta M=$ $\delta(\operatorname{co}(M))$.

By combining the last corollary with Lemma 6, we obtain the following result.

Corollary 12. For a field $\mathbb{F}$, let $(M, \varphi)$ be a connected $\mathbb{F}$-represented matroid for which $r^{*}(M)=2$. Then $\operatorname{co}(M) \cong U_{n-2, n}$ for some $n \geqslant 3$ and $\delta M \cong U_{2, n}$.

We observe that the connected matroids $M$ for which $\operatorname{co}(M) \cong U_{1,3}$ coincide with the cycle matroids of theta graphs.

\section{Connected matroids}

In this section, we prove some results that will be used in the proofs of the main theorems.

Lemma 13. Let e be an element of a connected matroid $M$. Then $M$ has at least $r^{*}(M)$ circuits containing e.

Proof. The result is true if $M$ is a circuit or a coloop and so holds if $|E(M)| \in\{1,2\}$. Assume the result is true for $|E(M)|<k$ and let $|E(M)|=k \geqslant 3$. Suppose $f \in E(M)-e$. Assume $M / f$ is connected. Then, by the induction assumption, as $r^{*}(M / f)=r^{*}(M)$, we see that $M / f$, and hence $M$, has at least $r^{*}(M)$ circuits containing $e$. We may now assume that $M / f$ is disconnected. Then $M \backslash f$ is connected and so has at least $r^{*}(M \backslash f)$ circuits containing $e$. As the connected matroid $M$ certainly has a circuit containing $\{e, f\}$, we deduce that $M$ has at least $r^{*}(M \backslash f)+1$, that is, $r^{*}(M)$, circuits containing $e$. The result follows by induction. 
Lemma 14. Let $M$ be a nonempty connected matroid. Then

$$
|\mathcal{C}(M)| \geqslant\left(\begin{array}{c}
r^{*}(M)+1 \\
2
\end{array}\right) \quad \text { and } \quad r^{*}(\delta M) \geqslant\left(\begin{array}{c}
r^{*}(M) \\
2
\end{array}\right) .
$$

Proof. We prove the first inequality by induction on $|E(M)|$. The result clearly holds for $r^{*}(M)=1$ and so holds for $|E(M)| \in\{1,2\}$. Assume it holds for $|E(M)|<k$ and let $|E(M)|=k \geqslant 3$. Take $e$ in $E(M)$. Suppose $M \backslash e$ is connected. Then, by the induction assumption,

$$
|\mathcal{C}(M \backslash e)| \geqslant\left(\begin{array}{c}
r^{*}(M \backslash e)+1 \\
2
\end{array}\right)=\left(\begin{array}{c}
r^{*}(M) \\
2
\end{array}\right) .
$$

By Lemma $13, M$ has at least $r^{*}(M)$ circuits containing $e$. Thus

$$
|\mathcal{C}(M)| \geqslant\left(\begin{array}{c}
r^{*}(M) \\
2
\end{array}\right)+\left(\begin{array}{c}
r^{*}(M) \\
1
\end{array}\right)=\left(\begin{array}{c}
r^{*}(M)+1 \\
2
\end{array}\right) .
$$

We may now assume that $M \backslash e$ is disconnected. Then $M / e$ is connected, so

$$
|\mathcal{C}(M)| \geqslant|\mathcal{C}(M / e)| \geqslant\left(\begin{array}{c}
r^{*}(M / e)+1 \\
2
\end{array}\right)=\left(\begin{array}{c}
r^{*}(M)+1 \\
2
\end{array}\right) .
$$

The first inequality follows by induction. The second inequality is a straightforward consequence of the first since, by Corollary $4,|\mathcal{C}(M)|=r^{*}(\delta M)+r^{*}(M)$.

We omit the proof of the following straightforward consequence of the last lemma.

Corollary 15. Let $M$ be a connected matroid. Then

$$
|\mathcal{C}(M)| \geqslant 3\left(r^{*}(M)-1\right) .
$$

For a matroid $M$ and a basis $B$ of $M$, the graph $G_{B}(M)$ is the simple bipartite graph having $B$ and $E(M)-B$ as its vertex classes where a vertex $x$ of $E(M)-B$ is adjacent to a vertex $y$ of $B$ if and only if $y \in C(x, B)$. Cunningham [2] and Krogdahl [4] proved the following result (see also [6, Proposition 4.3.2]).

Lemma 16. The vertex sets of the components of the graph $G_{B}(M)$ coincide with the components of the matroid $M$.

Lemma 17. Let $(M, \varphi)$ be an $\mathbb{F}$-represented matroid such that $M$ has no coloops. If $M=M_{1} \oplus M_{2}$, then $\delta M=\delta M_{1} \oplus \delta M_{2}$.

Proof. For a basis $B$ of $M$, we know that $\delta M$ is spanned by the set $\{C(f, B): f \in E-B\}$ of fundamental circuits of $B$. By Lemma 16, the components of $G_{B}(M)$ coincide with the components of $M$. In particular, if $B_{i}=E\left(M_{i}\right) \cap B$ for each $i$, then $\left\{C\left(f, B_{i}\right): f \in\right.$ $\left.E\left(M_{i}\right)-B_{i}\right\}$ spans $\delta M_{i}$. Since $M$ has no circuit that meets both $E\left(M_{1}\right)$ and $E\left(M_{2}\right)$, it follows that no circuit of $\delta M$ meets both $E\left(\delta M_{1}\right)$ and $E\left(\delta M_{2}\right)$. We deduce that $\delta M=$ $\delta M_{1} \oplus \delta M_{2}$. 
Theorem 18. Let $(M, \varphi)$ be an $\mathbb{F}$-represented matroid with no coloops. If $\delta M=N_{1} \oplus N_{2}$, then there are matroids $M_{1}$ and $M_{2}$ such that $M=M_{1} \oplus M_{2}$ and $N_{i}=\delta M_{i}$ for each $i$.

Proof. Let $E(M)=\left\{e_{1}, e_{2}, \ldots, e_{m}\right\}$ where $\left\{e_{1}, e_{2}, \ldots, e_{r}\right\}$ is a basis $B$ of $M$. Then $\delta M$ is spanned by $\left\{C\left(e_{r+1}, B\right), C\left(e_{r+2}, B\right), \ldots, C\left(e_{m}, B\right)\right\}$. We may assume that $N_{1}$ and $N_{2}$ are spanned by $\left\{C\left(e_{i}, B\right): r+1 \leqslant i \leqslant s\right\}$ and $\left\{C\left(e_{i}, B\right): s+1 \leqslant i \leqslant m\right\}$, respectively. Let $E_{1}=\cup_{i=r+1}^{s} C\left(e_{i}, B\right)$ and $E_{2}=\cup_{i=s+1}^{m} C\left(e_{i}, B\right)$.

Suppose $E_{1} \cap E_{2} \neq \varnothing$. Then we may assume that $C\left(e_{r+1}, B\right) \cap C\left(e_{m}, B\right) \neq \varnothing$. Then $M$ has a circuit $C$ containing $\left\{e_{r+1}, e_{m}\right\}$. Writing the circuit vector $\mathbf{c}_{C}$ as a linear combination of the circuit vectors $\mathbf{c}_{r+1}, \mathbf{c}_{r+2}, \ldots, \mathbf{c}_{m}$ where $\mathbf{c}_{j}$ is the circuit vector of $C\left(e_{j}, B\right)$, we see that the coefficients of $\mathbf{c}_{r+1}$ and $\mathbf{c}_{m}$ must both be non-zero. We deduce that, in $\delta M$, there is a circuit containing the elements $C\left(e_{r+1}, B\right)$ and $C\left(e_{m}, B\right)$. This is a contradiction as these elements are in different components of $\delta M$. We conclude that $E_{1} \cap E_{2}=\varnothing$.

Because $M$ has no coloops, every element of $M$ is in $E_{1}$ or $E_{2}$. Letting $M_{i}=M \mid E_{i}$, we see that $\delta M_{i}=N_{i}$.

Corollary 19. For a connected representable matroid $M$ and all $k \geqslant 0$, the matroid $\delta^{k} M$ is connected and

$$
r^{*}\left(\delta^{k} M\right) \geqslant 2^{k}\left(r^{*}(M)-3\right)+3 .
$$

Proof. We argue by induction on $k$. The result is immediate if $k=0$. Now assume the result holds for $k-1$, which is non-negative. By Corollaries 4 and 15,

$$
r^{*}\left(\delta^{k} M\right)+r^{*}\left(\delta^{k-1} M\right)=\left|\mathcal{C}\left(\delta^{k-1} M\right)\right| \geqslant 3\left(r^{*}\left(\delta^{k-1} M\right)-1\right) .
$$

Thus $r^{*}\left(\delta^{k} M\right) \geqslant 2 r^{*}\left(\delta^{k-1} M\right)-3$. Hence, by the induction assumption, as $\delta^{k-1} M$ is connected, it is either a coloop or has no coloops. Using Theorem 18, we see that, in each case, $\delta^{k} M$ is connected. Moreover, by the induction assumption again,

$$
r^{*}\left(\delta^{k} M\right) \geqslant 2\left(2^{k-1}\left(r^{*}(M)-3\right)+3\right)-3=2^{k}\left(r^{*}(M)-3\right)+3 .
$$

Thus the result holds by induction.

\section{The classification of derived sequences}

In this section, we classify derived sequences into finite, cyclic, and divergent types, and characterize each of them. By Theorem 18, we may focus on connected matroids.

Lemma 20. Let $(M, \varphi)$ be a connected $\mathbb{F}$-represented matroid. Then

(i) $\delta M \cong U_{0,0}$ if and only if $M \cong U_{1,1}$;

(ii) $\delta M \cong U_{1,1}$ if and only if $M \cong U_{n, n+1}$ for some $n \geqslant 0$; and

(iii) $\delta M \cong U_{n, n+1}$ for some $n \geqslant 0$ if and only if $n=2$ and $M \cong M(G)$ where $G$ is a theta graph. 
Proof. Parts (i) and (ii) are straightforward to check. Now assume that $\delta M \cong U_{n, n+1}$ for some $n \geqslant 0$. Then, by Corollary 4, $n+1=r^{*}(M)+r^{*}(\delta M)=r^{*}(M)+1$, so $r^{*}(M)=n$. As $\delta M$ is simple, $n \geqslant 2$. By Corollary $15,|\mathcal{C}(M)| \geqslant 3\left(r^{*}(M)-1\right)$, so $r^{*}(M)+1 \geqslant 3\left(r^{*}(M)-1\right)$. Hence $r^{*}(M) \leqslant 2$. Thus $r^{*}(M)=2$, so, by Corollary 12 , for some $t \geqslant 3$, we have that $\operatorname{co}(M) \cong U_{t-2, t}$ and $\delta M \cong U_{2, t}$. We deduce that $n=2$ and $t=3$. Thus $M \cong M(G)$ where $G$ is a theta graph. The converse is established in Corollary 12.

Lemma 21. Let $(M, \varphi)$ be a connected $\mathbb{F}$-represented matroid. Then $\delta M$ is not the cycle matroid of a theta graph.

Proof. Suppose $\delta M$ is the cycle matroid of a theta graph. Then $r^{*}(\delta M)=2$. Thus, by Corollary 4, $|\mathcal{C}(M)|=r^{*}(M)+2$. But, by Corollary $15,|\mathcal{C}(M)| \geqslant 3\left(r^{*}(M)-1\right)$. Hence $r^{*}(M) \leqslant 2$. By Lemma $20, r^{*}(M) \notin\{0,1\}$. Thus $r^{*}(M)=2$, so, by Corollary 12 , $\delta M \cong U_{2, n}$ for some $n \geqslant 3$, a contradiction.

Lemma 22. Let $M$ be a 6 -element rank-3 simple matroid. Then $|\mathcal{C}(M)| \geqslant 7$ with equality if and only if $M \cong M\left(K_{4}\right)$.

Proof. If $M$ is not 3-connected, then it is either the 2-sum of $U_{2,3}$ and $U_{2,5}$ or the parallel connection of $U_{2,3}$ and $U_{2,4}$. One easily checks that, in these cases, $|\mathcal{C}(M)|=10$ and $|\mathcal{C}(M)|=8$, respectively. If $M$ is 3-connected, then $M$ is isomorphic to $M\left(K_{4}\right), \mathcal{W}^{3}, Q_{6}$, $P_{6}$, or $U_{3,6}$ (see, for example, [6, Corollary 12.2.19]) where each of the last four matroids is obtained from its predecessor by relaxing a circuit-hyperplane. Since each such relaxation eliminates one circuit but adds $r^{*}$ new circuits, we deduce that $|\mathcal{C}(M)| \geqslant \mid \mathcal{C}\left(M\left(K_{4}\right) \mid\right.$ with equality if and only if $M \cong M\left(K_{4}\right)$. As $M\left(K_{4}\right)$ has exactly seven circuits, the lemma follows

The next theorem answers Longyear's Questions 3(a) and 3(c) for represented matroids over arbitrary fields. In particular, Theorem 1 is an immediate consequence of this result.

Theorem 23. Let $(M, \varphi)$ be a nonempty $\mathbb{F}$-represented matroid. If $\delta^{k} M \cong M$ for some $k \geqslant 1$, then $M$ is a direct sum of matroids each of which is isomorphic to $U_{2,4}$.

Proof. It suffices to show that if $\delta^{k} M \cong M$ for some $k \geqslant 1$ and $M$ is connected, then $M \cong U_{2,4}$. Thus we assume that $M$ is connected. We have $r^{*}(M)=r^{*}\left(\delta^{k} M\right)$, so, by Corollary 19,

$$
r^{*}(M)=r^{*}\left(\delta^{k} M\right) \geqslant 2^{k}\left(r^{*}(M)-3\right)+3 .
$$

Hence $0 \geqslant\left(2^{k}-1\right)\left(r^{*}(M)-3\right)$, so $r^{*}(M) \leqslant 3$. By Lemma 20, $r^{*}(M) \notin\{0,1\}$.

Suppose $r^{*}(M)=3$. Then $r^{*}\left(\delta^{k} M\right)=3$. As $r^{*}\left(\delta^{i} M\right) \geqslant 2 r^{*}\left(\delta^{i-1} M\right)-3$ for all $i$ in $[k]$, it follows by induction that $r^{*}\left(\delta^{i} M\right)=3$ for all such $i$. Since $r\left(\delta^{k} M\right)=r^{*}\left(\delta^{k-1} M\right)$, we deduce that $r(M)=3$. Hence $M$ is a 6 -element rank-3 matroid having exactly six circuits. As $\delta^{k} M$ is simple, so is $M$ and we have a contradiction to Lemma 22. Thus $r^{*}(M) \neq 3$, so $r^{*}(M)=2$. 
Now $r(\delta M)=r^{*}(M)=2$. Since $\delta M$ is simple, it follows that $\delta M \cong U_{2, n}$ for some $n \geqslant 3$. If $n=3$, then $\delta M, \delta^{2} M$, and $\delta^{3} M$ are $U_{2,3}, U_{1,1}$, and $U_{0,0}$, so, for all $i \geqslant 1$, no $\delta^{i} M$ is isomorphic to $M$. If $n \geqslant 5$, then $r^{*}(\delta M) \geqslant 3>r^{*}(M)$, so $k \geqslant 2$. Then

$$
r^{*}\left(\delta^{k} M\right) \geqslant 2^{k-1}\left(r^{*}(\delta M)-3\right)+3 \geqslant 3>r^{*}(M),
$$

a contradiction. We conclude that $n=4$, that is, $\delta M \cong U_{2,4}$. By Lemma $6, \delta^{k} M \cong U_{2,4}$ for all $k \geqslant 1$, so $r\left(\delta^{k} M\right)=r^{*}\left(\delta^{k-1} M\right)=2$. Hence $r(M)=2$, so $M$ is a simple 4-element matroid of rank two, that is, $M \cong U_{2,4}$.

Theorem 24. Let $M$ be a connected represented matroid that is not isomorphic to $U_{0,0}$, $U_{1,1}$, a circuit, the cycle matroid of a theta graph, or a matroid whose cosimplification is $U_{2,4}$. Then, for all $k \geqslant 1$,

$$
r^{*}\left(\delta^{k+1} M\right)>r^{*}\left(\delta^{k} M\right) .
$$

Moreover, $r^{*}\left(\delta^{k} M\right) \geqslant 2^{k-1}+3$ unless $M \cong U_{1,4}$. In the exceptional case, $r^{*}(M)=3=$ $r^{*}(\delta M)$ and $r^{*}\left(\delta^{2} M\right)=4$, so $r^{*}\left(\delta^{k} M\right) \geqslant 2^{k-2}+3$ for all $k \geqslant 2$.

Proof. We may assume that $r^{*}(M) \geqslant 2$ otherwise $M$ is $U_{0,0}, U_{1,1}$, or a circuit. By Corollary 19,

$$
r^{*}\left(\delta^{k+1} M\right) \geqslant 2 r^{*}\left(\delta^{k} M\right)-3 .
$$

Then, for $k \geqslant 1$,

$$
r^{*}\left(\delta^{k} M\right) \geqslant 2^{k-1}\left(r^{*}(\delta M)-3\right)+3 \geqslant 2^{k-1}\left(2 r^{*}(M)-6\right)+3 .
$$

Thus $r^{*}\left(\delta^{k} M\right) \geqslant 2^{k-1}+3 \geqslant 4$ provided $r^{*}(\delta M) \geqslant 4$ or $r^{*}(M) \geqslant 4$. In each of these cases, the lemma holds since, by $(1), r^{*}\left(\delta^{k+1} M\right)>r^{*}\left(\delta^{k} M\right)+r^{*}\left(\delta^{k} M\right)-4 \geqslant r^{*}\left(\delta^{k} M\right)$.

We may now assume that $r^{*}(\delta M)<4$ and $r^{*}(M)<4$. Suppose $r^{*}(M)=3$. Then $r(\delta M)=3$. As $r^{*}(\delta M) \leqslant 3$, we see that $|E(\delta M)| \leqslant 6$, that is, $|\mathcal{C}(M)| \leqslant 6$. Hence, by Lemma $22,|E(M)| \leqslant 5$. Suppose $|E(M)|=4$. Then $M \cong U_{1,4}$ and, by Lemma 7 , $\delta M \cong M\left(K_{4}\right)$. Thus $r^{*}(\delta M)=3$ and $r^{*}\left(\delta^{2} M\right)=4$. Therefore the first inequality in the lemma holds when $k=1$. For $k \geqslant 2$, we have $r^{*}\left(\delta^{k} M\right) \geqslant 2^{k-2}\left(r^{*}\left(\delta^{2} M\right)-3\right)+3$ and, using (1), we see that the lemma follows when $M \cong U_{1,4}$ since $r^{*}\left(\delta^{2} M\right)=4$.

Now suppose that $r^{*}(M)=3$ and $|E(M)|=5$. Then $M$ is isomorphic to $U_{2,5}$ or the matroid that is obtained from $U_{2,4}$ by adding an element in parallel to an existing element. In each of these cases, $|\mathcal{C}(M)| \geqslant 8$, so $r^{*}(\delta M) \geqslant 5$, a contradiction.

We may now assume that $r^{*}(M)=2$ and $r^{*}(\delta M) \leqslant 3$. Then $r(\delta M)=2$ and, by Corollary $12, \delta M \cong U_{2,4}$. Since $M^{*}$ is a rank-2 connected matroid with exactly four rank-one flats, we deduce that $\operatorname{co}(M) \cong U_{2,4}$, a contradiction.

We now answer Longyear's Question 4 for arbitrary represented matroids and thereby prove Theorem 2 .

Theorem 25. Let $M$ be a represented matroid such that $\delta^{k} M \cong U_{0,0}$ for some $k \geqslant 0$. Then $\delta^{3} M \cong U_{0,0}$ and each component of $M$ is isomorphic to $U_{1,1}$, a circuit, or the cycle matroid of a theta graph. 
Proof. By Lemma 20, if each component of $M$ is isomorphic to $U_{1,1}$, a circuit, or the cycle matroid of a theta graph, then $\delta^{3} M \cong U_{0,0}$. Now let $N$ be a component of $M$. By Lemma 6 , if $\operatorname{co}(N) \cong U_{2,4}$, then $\delta^{k} M$ has $U_{2,4}$ as a component for all $k \geqslant 1$, so $\delta^{k} M \nsubseteq U_{0,0}$. We may now assume that $N$ is not isomorphic to $U_{1,1}$, a circuit, the cycle matroid of a theta graph, or a matroid whose cosimplification is $U_{2,4}$ Then, for all $k \geqslant 1$, by Theorem $24, r^{*}\left(\delta^{k} M\right) \geqslant 3$, so $\delta^{k} M \nsubseteq U_{0,0}$.

\section{References}

[1] T. H. Brylawski and D. Lucas. Uniquely representable combinatorial geometries. In Teorie combinatorie (Proc. 1973 Internat. Colloq.), pages 83-104. Accademia Nazionale dei Lincei, Rome, 1976.

[2] W. H. Cunningham. A Combinatorial Decomposition Theory, Ph.D. thesis, University of Waterloo, 1973.

[3] J. Kahn. On the uniqueness of matroid representations over GF(4). Bull. London Math. Soc., 20:5-10, 1988.

[4] S. Krogdahl. The dependence graph for bases in matroids. Discrete Math., 19:47-59, 1977.

[5] J. Q. Longyear. The circuit basis in binary matroids. J. Number Theory, 12:71-76, 1980.

[6] J. Oxley. Matroid Theory, Second edition, Oxford University Press, New York, 2011.

[7] A. Recski. Matroids and electrical networks: A tribute to Masao Iri. In Proc. 11th Japanese-Hungarian Symposium on Discrete Mathematics and its Applications, pages 68-78. University of Tokyo Press, Tokyo, 2019. 